\title{
Application of the artificial neural network to optimize the formulation of self-nanoemulsifying drug delivery system containing
} rosuvastatin

\author{
Giang Thi Thu Vu ${ }^{1 *}$, Nghia Thi Phan², Huyen Thi Nguyen³, Hung Canh Nguyen', Yen Thi Hai Tran², Tung Bao Pham², Linh \\ Tran Nguyen ${ }^{1}$, Hoa Dang Nguyen ${ }^{1}$ \\ 'Department of Pharmaceutics, Hanoi University of Pharmacy, Hanoi, Vietnam. \\ ${ }^{2}$ Bioequivalence Centre, National Institute of Drug Quality Control, Hanoi, Vietnam. \\ ${ }^{3}$ School of Medicine and Pharmacy, Vietnam National University, Hanoi, Vietnam.
}

\section{ARTICLE INFO \\ Received on: 27/03/2020 \\ Accepted on: 31/07/2020 \\ Available online: 05/09/2020}

\section{Key words:}

Rosuvastatin, self-

nanoemulsifying

drug delivery system,

nanoemulsion, droplet size,

polydispersity index (PDI),

artificial neural network.

\begin{abstract}
The objectives of this study were to optimize the formula of the self-nanoemulsifying drug delivery system (SNEDDS) containing rosuvastatin and to evaluate its physicochemical characteristics. The solubility and compatibility of rosuvastatin in surfactants, cosurfactants, and oil excipients were evaluated. The D-optimal experimental design, created by JMP 15 software, was used for analyzing the effects of excipients on the physicochemical characteristics of SNEDDS to optimize the rosuvastatin SNEDDS formula. The generated nanoemulsions from Ros SNEDDS were characterized for droplet size, polydispersity index, and entrapment efficiency. As a result, Cremophor RH40, Capryol 90, and PEG 400 were selected to develop the pseudoternary phase diagram to identify the area capable of selfforming nanoemulsion. As the percentage of rosuvastatin calcium increased from $8 \%$ to $12 \%$, the area for optimizing the formula of Ros SNEDDS decreased. The Ros SNEDDS prepared according to predicted formulas possessed selfemulsification to form nanoemulsion with average droplet size less than $100 \mathrm{~nm}$, polydispersity index less than 0.3 , and rosuvastatin entrapment higher than $90 \%$.
\end{abstract}

\section{INTRODUCTION}

Rosuvastatin is a hydroxymethylglutaryl coenzyme A reductase inhibitor. Rosuvastatin is used to reduce low density lipoprotein cholesterol, apolipoprotein B, and triglycerides and to increase high density lipoprotein cholesterol in the hyperlipidemias treatment, including primary hypercholesterolemia, mixed dyslipidemia, and hypertriglyceridemia, as well as in patients with homozygous familial hypercholesterolemia. Rosuvastatin can also be used to reduce the progression of atherosclerosis. However, one of the main limitations of rosuvastatin is the incomplete absorption from the gastrointestinal tract (Schachter, 2005). The absolute

${ }^{*}$ Corresponding Author

Giang Thi Thu Vu, Department of Pharmaceutics, Hanoi University of Pharmacy, Hanoi, Vietnam.E-mail: giangvtt@hup.edu.vn bioavailability of rosuvastatin is about $20 \%$ mainly due to its low solubility in water (Luvai et al., 2012; Olsson et al., 2006). In general, to enhance the bioavailability of drugs in biopharmaceutical classification system II classification, various methods can be used to improve the dissolution rate of the poorly water-soluble drugs. These methods have been extensively reported and reviewed, namely particle size reduction, nanonization (Chen et al., 2011), cosolvency, hydrotropy, sonocrystallization (Kawabata et al., 2011), inclusion into cyclodextrin (Das et al., 2018), solid dispersion (Baek et al., 2012), self-emulsifying drug delivery systems (Baek et al., 2012), and so on. Some of these methods have been successfully applied to improve the solubility and oral bioavailability of rosuvastatin. In primary approaches, rosuvastatin's solubility, dissolution rate, and bioavailability can be enhanced by decreasing the particle size or nanonization, for example, nanoparticle (Alshora et al., 2018) and nanocrystallization (Palani et al., 2015). In different approaches, rosuvastatin can be formulated into different carriers, such as 
$\beta$-cyclodextrin complexation (Kapse et al., 2016; Sarfraz et al., 2017), cyclodextrin-based nanosponges (Gabr et al., 2018), natural polymers (Kumar Katual et al., 2020), and solid dispersion systems (Mounica et al., 2015). Recently, nanostructured lipid carriers (solid lipid, SNEDDS, and SMEDDS) have been used and they show promising results in improving the solubility of rosuvastatin (Kamble et al., 2014; Li et al., 2018; Rizwanullah et al., 2017).

Self-nanoemulsifying drug delivery systems are anhydrous homogenous liquid mixtures consisting of oils, surfactants, cosurfactants, and drug substances, which would spontaneously form oil-in-water nanoemulsion (of approximately $200 \mathrm{~nm}$ or less in droplet size) upon dilution with water under gentle stirring. This characteristic makes SNEDDS an excellent approach for oral delivery of poorly soluble drugs with appropriate solubility in oil and/or surfactant mixtures (Kassem et al., 2017). After self-nanoemulsification in the biological fluid, small oil droplets of less than $5 \mu \mathrm{m}$ containing drug help enhance drug dissolution rate (Verma et al., 2014). Additionally, SNEDDS improves stability of drug molecules and enables the possibility of encapsulating the drug in gelatin capsules (Wang et al., 2009). Compared with conventional nanoemulsion, SNEDDS advantages are improved physical and/or chemical stability as well as the commercial viability and patient compliance/tolerability (Chen et al., 2011). Various studies have shown that oral bioavailability of several low solubility drugs can be enhanced when formulated as SNEDDS (Hong et al., 2006; Kumar Mantri, 2012; Mahmoud et al., 2013; Reddy, 2018). Therefore, our objectives in this study were to optimize the formula of SNEDDS containing rosuvastatin and evaluate its physicochemical characteristics.

\section{MATERIALS AND METHODS}

\section{Materials}

Rosuvastatin calcium (Ros) was obtained from Enaltec (India). Transcutol HP, Labrafil M1994 CS, and Capryol 90 were supplied by Gattefossé (France). Cremophor RH 40, Tween 20, Tween 80, and polyethylene glycol 400 (PEG 400) were purchased from BASF (Germany). Miglyol was supplied by Cremer OLEO GmbH \& Co (Germany); high-performance liquid chromatograph (HPLC)-grade acetonitrile and methanol were purchased from Fisher (USA). Water was purified by reverse osmosis in-house system. Other reagents were of analytical grade and purchased from Beijing Chemical Reagent Factory (China).

\section{Methods}

\section{Determination of the solubility of rosuvastatin in excipients}

The solubility of Ros in different excipients, including surfactants, cosurfactants, and oil phase, was determined as follows: excess amount of Ros was added to $5 \mathrm{ml}$ excipients in test tubes and then mixed by vortex (IKA ${ }^{\circledR}$ Vortex 3, SigmaAldrich) for 10 minutes to dissolve the active ingredient. After closing the test tubes, the mixtures were shaken in a reciprocal shaking and heating bath (BS-06/11/21/31, Jeiotech, Korea) at $30^{\circ} \mathrm{C}$ for 48 hours. All the mixtures maintained a persistent cloudy appearance or visible grains of solid were found deposited at the bottom of the test tube after being shaken. The test tubes were centrifuged at 3,000 rpm for 10 minutes. The supernatant was withdrawn and filtered through $0.45 \mu \mathrm{m}$ cellulose acetate membrane. The filtered solution was diluted by methanol and quantified by the HPLC method described in "Characterization of rosuvastatin SNEDDS".

\section{Compatibility of rosuvastatin with surfactant, cosurfactant, and oil phase}

Solutions of $3 \mathrm{mg} / \mathrm{ml}$ rosuvastatin in surfactant, cosurfactant, and oil excipients were stored in closed borosilicate glass bottles in a long-term condition $\left(30^{\circ} \mathrm{C} \pm 2{ }^{\circ} \mathrm{C}\right.$, $75 \% \pm 5 \% \mathrm{RH})$ and accelerated condition $\left(40^{\circ} \mathrm{C} \pm 2{ }^{\circ} \mathrm{C}, 75 \% \pm\right.$ $5 \% \mathrm{RH})$. The drug content in the solutions at each interval time was quantified by the HPLC method to reveal the influence of surfactants, cosurfactants, and oil excipients on rosuvastatin chemical stability for 3 months and at monthly intervals.

\section{Establishment of the pseudoternary phase diagram}

The pseudoternary phase diagram of Ros nanoemulsion was developed and the nanoemulsion region was identified by the characteristic of the droplet size. To obtain the nanoemulsion, droplet size must be less than $200 \mathrm{~nm}$ with polydispersity index of less than 0.3 after emulsifying the mixtures of surfactant, cosurfactant, and oil excipients with a constant ratio of water. The mixtures were prepared by adding appropriate amounts of surfactant, cosurfactant, and oil phase of different weight ratios in a stoppered test tube and vortexed vigorously to ensure thorough mixing. The mixture of surfactant and cosurfactant $\left(S_{\text {mix }}\right)$ was at different weight ratios: $4: 1,3: 1,2: 1,1: 1,1: 2,1: 3$, and 1:4. Next, the oil phase was added to the $S_{\text {mix }}$ at the weight ratio of $1: 9,2: 8$, $3: 7,4: 6,5: 5,6: 4,7: 3,8: 2$, and 9:1, respectively. After being well mixed, the obtained mixtures were emulsified with the triple amount of purified water by stirring (IKA magnetic stirrer) at 50 rpm for 3 minutes. The ternary phase diagram was plotted using the CHEMIX School v.7.0 software.

\section{Preparation of SNEDDS containing rosuvastatin}

Rosuvastatin calcium was dissolved in the mixture of surfactant, cosurfactant, and oil excipient by stirring at $100 \mathrm{rpm}$ at $50^{\circ} \mathrm{C}$ until a clear solution was achieved.

\section{Characterization of rosuvastatin SNEDDS}

Determination of the amount of rosuvastatin calcium: Ros was determined by a validated method using HPLC system (Shimadzu, Japan, Nexera XR) with the condition of Agilent C18 column, size $4.6 \times 250 \mathrm{~mm}$, and particle size $5 \mu \mathrm{m}$; mobile phase: a mixture of acetonitrile, $1 \%$ trifluoroacetic acid solution, and water with a volume ratio of 37:1:62, respectively; flow speed: 1.2 $\mathrm{ml} /$ minutes; injection sample volume: $20 \mu \mathrm{l}$; temperature: $40^{\circ} \mathrm{C}$; $\mathrm{UV}$ detector wavelength at $242 \mathrm{~nm}$.

Emulsification of Ros SNEDDS: about $1.4 \mathrm{~g}$ of Ros SNEDDS sample was emulsified with $5 \mathrm{ml}$ of water under the condition of magnetic stirring at $50 \mathrm{rpm}$ for 3 minutes.

Determination of droplet size and polydispersity index (PDI): the droplet size and PDI of emulsified Ros nanoemulsion were determined by the dynamic light scattering method (Malvern Panalytical, UK, Zetasizer ZS90). The Ros nanoemulsion was diluted with purified water and was filtered through a $0.2-\mu \mathrm{m}$ cellulose acetate membrane to ensure that the count rate was in the range of 200 and $400 \mathrm{kcps}$. 
Determination of entrapment efficiency of rosuvastatin: the obtained Ros nanoemulsion was diluted with water in a $10-\mathrm{ml}$ volumetric flask. $3.0 \mathrm{ml}$ of this emulsion was transferred into a 10,000 NMWL-Amicon Ultra-4 centrifugal filter. The water phase was separated by centrifugation at the speed of $2,000 \mathrm{rpm}$. The amount of drug in the SNEDDS $\left(X_{\text {total }}\right)$ and the water phase $\left(X_{\text {water }}\right)$ was determined. The rosuvastatin entrapment efficiency (EE) was calculated by the following equation:

$$
\mathrm{EE}=\frac{\left(\mathrm{X}_{\text {total }}-\mathrm{X}_{\text {water }}\right)}{\mathrm{X}_{\text {total }}} \times 100
$$

Robustness to centrifugation: the robustness of Ros SNEDDS to centrifugation was studied at 5,000 rpm for 30 minutes. Then, the nanoemulsion samples were observed for any signs of phase separation or precipitation.

\section{Experimental design and optimizing SNEDDS formulas}

D-optimal design created by JMP 15 software (SAS Institute Inc., Cary, NC) was applied to formulate Ros SNEDDS compositions.

Based on the artificial neural network, the effects of the input variables on output variables were also analyzed and the formula of Ros SNEDDS was optimized using JMP 15 software.

Table 1. Solubility of rosuvastatin in oil excipients, surfactants, and cosurfactants $(n=3)$

\begin{tabular}{llc}
\hline Groups & Excipients & Solubility $(\mathbf{m g} / \mathbf{m l})$ \\
\hline \multirow{4}{*}{ Oil } & Capryol 90 & $42.84 \pm 0.53$ \\
& Oleic acid & $28.65 \pm 0.09$ \\
& Miglyol & $1.57 \pm 0.06$ \\
& Isopropyl myristate & $0.49 \pm 0.04$ \\
Arachis oil & $10.40 \pm 1.72$ \\
Surfactants & Sunflower oil & $2.20 \pm 0.25$ \\
& Cremophor RH 40 & $100.65 \pm 0.77$ \\
& Labrafil & $33.11 \pm 0.25$ \\
Tween 20 & $99.25 \pm 0.47$ \\
Tween 80 & $99.94 \pm 0.96$ \\
Propylene glycol & $325.84 \pm 1.00$ \\
Polyethylene glycol 200 & $304.94 \pm 0.44$ \\
Polyethylene glycol 400 & $474.60 \pm 0.50$ \\
& Glycerol & $39.51 \pm 0.59$ \\
Ethanol & $5.23 \pm 0.01$ \\
& Transcutol HP & $361.49 \pm 0.48$ \\
\hline
\end{tabular}

\section{Data analysis and statistics}

A minimum of three replicates was performed for each experiment. The results are expressed as mean \pm standard deviation (SD). Computerized data were statistically described using Microsoft Excel v16.0 (Microsoft, USA).

\section{RESULTS AND DISCUSSION}

\section{Solubility of Ros in various excipients}

The solubility of Ros in various excipients including surfactants (Cremophor RH 40, Labrafil Tween 80, and Tween 20), cosurfactants (propylene glycol, PEG 200, PEG 400, glycerol, ethanol, and Transcutol HP), and oils (Capryol 90, oleic acid, Miglyol, isopropyl myristate, arachis oil, and sunflower oil) was determined as described in "Determination of the solubility of rosuvastatin in excipients." The results are shown in Table 1.

It was clear to find out that, among examined surfactants, cosurfactants, and oils, Cremophor RH 40, PEG 400, and Capryol 90, respectively, achieved the highest solubility of Ros. Appropriate excipients should have a good solubilizing capacity of the drug substance, which is essential to incorporate the drug into the solution. In addition, the chosen excipients also should not affect the stability of the drug substance. Thus, in this study, in each group, two excipients that dissolved the most of Ros were chosen. Their influences on the stability of Ros were evaluated consequently. The chosen excipients were Capryol 90 and oleic acid (oil group), Cremophor RH 40 and Tween 80 (surfactant group), and PEG 400 and Transcutol HP (cosurfactant group).

\section{Influences of surfactant, cosurfactant, and oil excipients on the chemical stability of Ros}

Influences of surfactant, cosurfactant, and oil excipients on the chemical stability of Ros were investigated to find out that the main components of SNEDDS could not only dissolve Ros completely but also did not affect its stability. Ros was separately dissolved in each selected excipient and then filled in the closed glass vial. The stability study was implemented according to the method described in "Compatibility of rosuvastatin with surfactant, co-surfactant and oil phase." The obtained results are presented in Tables 2 and 3.

After 3-month storage in the long-term condition, Ros content in the excipients, including Cremophor RH 40, Tween 80, Capryol 90, PEG 400, and Transcutol HP, changed insignificantly. However, when dissolved in oleic acid, the content of Ros decreased significantly to $86.02 \%$ after 1 month.

Table 2. Changes in rosuvastatin content when stored under long-term condition $(n=3)$.

\begin{tabular}{lcccc}
\hline \multirow{2}{*}{ Excipients } & \multicolumn{4}{c}{ Rosuvastatin content (\%) } \\
\cline { 2 - 5 } & Initial & After 1 month & After 2 months & After 3 months \\
\hline Cremophor RH 40 & $99.21 \pm 0.23$ & $98.68 \pm 0.98$ & $98.92 \pm 0.36$ & $98.10 \pm 0.67$ \\
Tween 80 & $102.11 \pm 2.45$ & $102.13 \pm 0.97$ & $103.48 \pm 1.07$ & $103.66 \pm 1.43$ \\
Capryol 90 & $101.22 \pm 1.32$ & $101.90 \pm 0.78$ & $100.96 \pm 1.14$ & $101.46 \pm 1.34$ \\
Oleic acid & $99.86 \pm 1.12$ & $86.02 \pm 0.98$ & - & - \\
PEG 400 & $101.82 \pm 1.89$ & $101.28 \pm 0.45$ & $102.24 \pm 0.32$ & $102.01 \pm 0.54$ \\
Transcutol HP & $98.52 \pm 1.45$ & $98.93 \pm 0.67$ & $97.18 \pm 0.92$ & $97.71 \pm 0.89$ \\
\hline
\end{tabular}


Table 3. Changes in rosuvastatin content when stored under accelerated condition $(n=3)$.

\begin{tabular}{lcccc}
\hline \multirow{2}{*}{ Excipients } & \multicolumn{4}{c}{ Rosuvastatin content (\%) } \\
\cline { 2 - 5 } & Initial & After 1 month & After 2 months & After 3 months \\
\hline Cremophor RH 40 & $99.21 \pm 0.23$ & $98.98 \pm 1.02$ & $99.72 \pm 0.78$ & $98.95 \pm 0.47$ \\
Tween 80 & $102.11 \pm 2.45$ & $102.33 \pm 0.86$ & $102.80 \pm 1.05$ & $102.36 \pm 1.40$ \\
Capryol 90 & $101.22 \pm 1.32$ & $100.90 \pm 0.90$ & $99.72 \pm 1.18$ & $99.08 \pm 0.68$ \\
Oleic acid & $99.86 \pm 1.12$ & - & - & - \\
PEG 400 & $101.82 \pm 1.89$ & $101.64 \pm 0.58$ & $101.02 \pm 0.80$ & $99.87 \pm 0.94$ \\
Transcutol HP & $98.52 \pm 1.45$ & $96.02 \pm 0.70$ & $96.88 \pm 1.30$ & $97.23 \pm 0.87$ \\
\hline
\end{tabular}

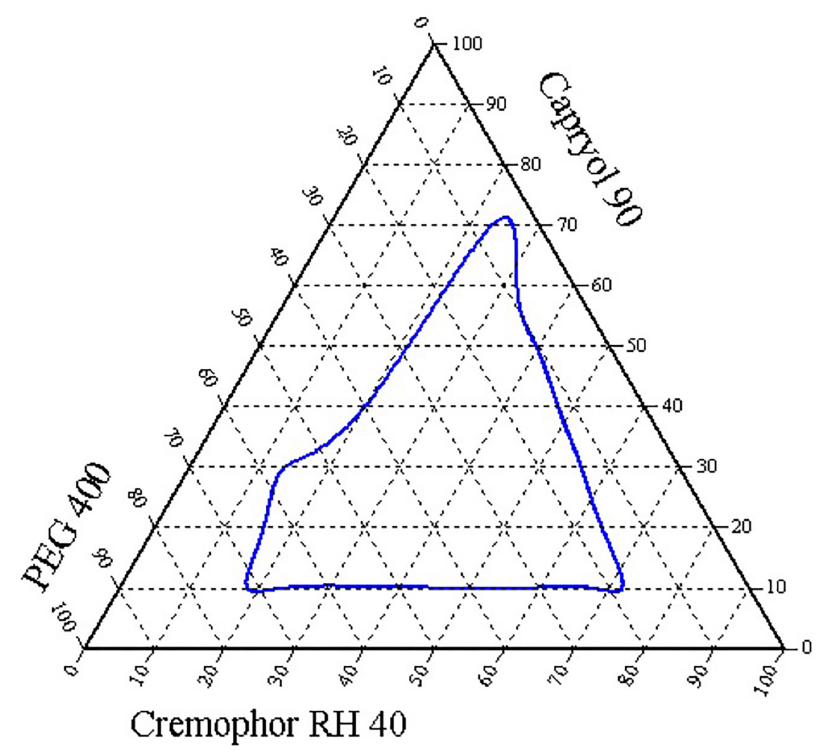

Figure 1. Pseudoternary phase diagram of SNEDDS (ternary mixture inside the solid line exhibited self-nanoemulsification).

Under the accelerated condition, Ros content in the prepared solutions was stable. However, not surprisingly, after one month, we could not detect the Ros in oleic acid.

In addition, no change in color or precipitation in the rosuvastatin solution in selected excipients was observed during 3 months of investigation of the stability in the long-term and accelerated condition, suggesting that there was no physical incompatibility.

Based on the requirements of dissolving capacity, safety, and compatibility with the active pharmaceutical ingredient, Cremophor RH 40, Capryol 90, and PEG 400 were selected and served as surfactant, cosurfactant, and oil excipient for Ros SNEDDS.

\section{Pseudoternary phase diagram}

Pseudoternary phase diagrams were used to identify the nanoemulsion forming area, which is shown in Figure 1 (the area defined by the solid line). The existing area of the selfnanoemulsification domain is represented by the plotted numbers that can form nanoemulsion.

The results demonstrated that Cremophor RH40, PEG 400, and Capryol 90 had a high self-nanoemulsification capacity.
A large self-nanoemulsification region was formed with the proportion of Capryol 90 in the range of $10 \%-70 \%$, PEG 400 in the range of $6 \%-72 \%$, and Cremophor $\mathrm{RH} 40$ in the range of $14 \%-72 \%$.

The phase diagram was constructed by three components: oil, surfactant, and cosurfactant. The region of forming the nanoemulsion was determined by assessing the droplet size of the emulsion formed after adding a fixed amount of water to the threecomponent mixture in the phase diagram. This method has been widely used and successfully applied in the previous studies of SNEDDS containing simvastatin (Mahmoud, 2013).

Capryol 90, Cremophor RH40, and PEG 400 were selected as the main components of SNEDDS in the role of oil excipients, surfactant, and cosurfactant, respectively. Our results were in correlation with the other results of drug solubility in these excipients. Among surfactants, rosuvastatin was dissolved well in Cremophor RH 40 (Abo Enin, 2015). Karasulu et al. (2018) also found out that Cremophor EL has the highest solubilizing capacity of rosuvastatin calcium compare to Labrasol, Labrafil M, Tween 80, and Span 80. Among the studied cosurfactants, the solubility of rosuvastatin in PEG 400 was the highest. In other researches, some studies have used the combination of PEG 200 and PEG 400 as cosurfactants, but in our study, PEG 400 showed more benefit. In addition, the compatibility of the selected excipients with the active ingredient was essential to ensure the stability of the achieved SNEDDS.

\section{The effect of the drug concentration on the characteristics of nanoemulsion}

Incorporation of the drug into the SNEDDS may have some effects on the self-emulsifying performance, so the effect of drug loading on the characteristics of nanoemulsion was studied.

Various concentrations (w/w) of Ros (namely 2\%, 4\%, $6 \%, 8 \%, 10 \%, 12 \%$, and $14 \%$ ) in the mixture of Capryol 90 Cremophor RH 40-PEG 400 were investigated. The proportion of $S_{\text {mix }}$ (surfactant:cosurfactant) and proportion of $\mathrm{O}: S_{\text {mix }}$ on these samples were 3:1 and 3:7, respectively.

The results showed that Ros was completely dissolved when its concentration was lower than $14 \%$. At the concentration of $14 \%$, precipitation of Ros was observed at the bottom of the vessel; therefore, only mixtures with Ros concentration from $2 \%$ to $12 \%$ were further evaluated. The mixtures were emulsified by adding a triple amount of purified water under the condition of stirring at $50 \mathrm{rpm}$ for 3 minutes. The obtained nanoemulsion was evaluated for several properties, including droplet size, PDI, and robustness. 
The effects of drug concentration on droplet size and PDI are shown in Figure 2. The results revealed that droplet size and PDI increased inversely proportional to the Ros concentration. All the emulsion samples had a droplet size below $100 \mathrm{~nm}$. However, only the samples with the Ros concentration from $8 \%$ to $12 \%$ possessed the PDI around the value of 0.3 .

Nanoemulsions from Ros with concentration from 2\% to $12 \%$ were stable under the impact of the centrifugal force at speed of 5,000 rpm for 30 minutes. Neither phase separation nor precipitation was observed.

\section{Influences of the excipients on Ros SNEDDS properties}

Based on the results of the studies of pseudoternary phase diagram construction and effects of drug concentration on characteristics of nanoemulsion, experimental design to formulate Ros SNEDDS compositions was implemented. The independent and dependent variables and the requirements are shown in Table 4.

The data in Table 5 show that the proportion of each ingredient of Ros SNEDDS has an effect on droplet size, PDI, and drug entrapment of the SNEDDS. All the SNEDDS rosuvastatin samples were formulated with different ingredients ratio in the formula (rosuvastatin, Cremophor RH40, Capryol 90, and PEG 400). After self-emulsification, each sample of emulsion was created with distinctive characteristics of droplet size, PDI, and drug entrapment. By using area optimizing, these characteristics were influenced in the harmonization of all variables, namely the percentage of Ros and the proportion of Cremophor RH40 and Capryol 90.

The effects of the input variables on output variables were analyzed and the SNEDDS formula was optimized using the JMP 15 software (SAS Institute Inc., Cary, NC) based on the artificial neural network. Output variables were separately trained by a three-node one-hidden layer neural network (Fig. 3).

The hyperbolic tangent function (TanH) was applied at each node of the hidden layer as the activation function. In the training process, the learning rate and number of tours were set to 0.1 and 100 , respectively. K-fold method was used to validate the training process. The original data in Table 5 were divided into $\mathrm{K}$ subsets $(\mathrm{K}=5)$. In turn, each of the $\mathrm{K}$ sets was used to validate the model fit on the rest of the data, fitting a total of $\mathrm{K}$ models. The model giving the best validation statistic was chosen as the final model. The training and validation results are shown in Table 6.

The R-square statistics for both training and validation sets were greater than 0.87 , signifying that the models were describing well the data used to train the models and predicting well the data not used to train the models.

Contour plots shown in Figure 4 describe the general understanding of how the input variables impact the output variables.

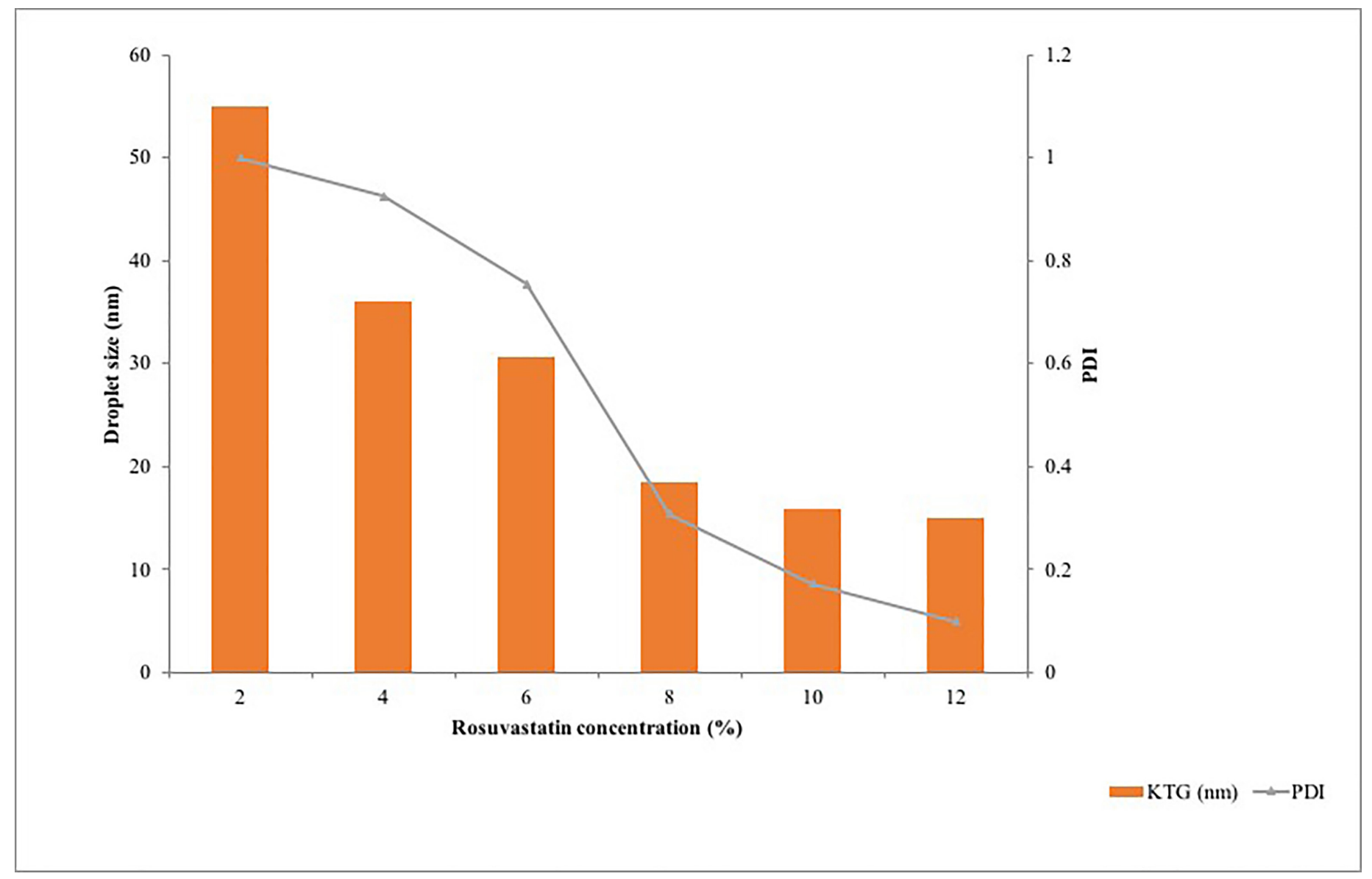

Figure 2. Droplet size and PDI of nanoemulsion prepared with various Ros concentrations. 
It was clear that the droplet size of nanoemulsion, PDI, and Ros entrapment were all affected by the proportion of Cremophor RH 40, PEG 400, and Capryol 90 in the SNEEDS formulas.

Contour plots in Figure 4A showed that when the percentage of Ros was $10 \%$ and the proportion of PEG 400 was in the range of $0.10-0.60$, the droplet size of nanoemulsion increased proportionally to the amount of Capryol 90, but inversely proportional to the amount of Cremophor RH40.

Table 4. Independent and dependent variables.

\begin{tabular}{llcc}
\hline \multirow{2}{*}{ Independent variables } & Type of variables & \multicolumn{2}{c}{ Levels } \\
\cline { 3 - 4 } & & Low & Height \\
\hline $\mathrm{X}_{1}=$ percentage of Ros (\%) & Quantitative & 8 & 12 \\
$\mathrm{X}_{2}=$ proportion of Cremophor RH40 & Formulation & 0.15 & 0.45 \\
$\mathrm{X}_{3}=$ proportion of Capryol 90 & Formulation & 0.10 & 0.50 \\
$\mathrm{X}_{4}=$ proportion of PEG 400 & Filler & \\
Dependent variables & Requirements & & \\
Droplet size (d.nm) & $\leq 100$ & & \\
PDI & $\leq 0.3$ & & \\
Ros entrapment $(\%)$ & $\geq 90$ & & \\
\hline
\end{tabular}

${ }^{\mathrm{a}} \mathrm{X}_{2}+\mathrm{X}_{3}+\mathrm{X}_{4}=1$
Contour plots in Figure 4B reveal that there are two areas where the PDI value is less than 0.3. The first area is determined by the proportion of PEG 400, Capryol 90, and Cremophor RH40 which were in the range of $0.10-0.20,0.35-0.50$, and $0.37-0.45$, respectively. In this area, the value of PDI increased proportionally to the amount of PEG 400 but inversely proportional to the amount of Capryol 90. The second area is determined by the proportion of PEG 400, Capryol 90, and Cremophor RH40 in the range of $0.31-0.60,0.10-0.30$, and $0.23-0.45$, respectively.

It can be seen in Figure $4 \mathrm{C}$ that when the proportion of PEG was in the range of $0.10-0.22$ or $0.25-0.60$, the Ros entrapment increased inversely proportional to the amount of Cremophor RH40 but proportional to the amount of Capryol 90 in the SNEDDS formula. Particularly, the Ros entrapment was constant at the value of $97 \%$ in the area determined by the proportion of PEG 400, Capryol 90, and Cremophor RH40 in the range of $0.22-0.25,0.32-0.41$, and $0.38-0.45$, respectively.

Figure 5 shows that the physicochemical properties of nanoemulsion are affected by the percentage of Ros. The droplet size and PDI of nanoemulsion increased proportionally to the amount of the drug substance, whereas drug entrapment in the nanoemulsion droplets was influenced by the drug load in a complex manner. When the percentage of Ros in the formulas

Table 5. Formulas and characteristics of Ros SNEDDS ( $n=3)$.

\begin{tabular}{cccccccc}
\hline Formulas & $\mathbf{X}_{1}$ & $\mathbf{X}_{2}$ & $\mathbf{X}_{3}$ & $\mathbf{X}_{4}$ & Droplet size (d.nm) & PDI & Ros entrapment (\%) \\
\hline F1 & 8 & 0.40 & 0.20 & 0.40 & 15.93 & 0.268 & 92.60 \\
F2 & 12 & 0.40 & 0.20 & 0.40 & 20.50 & 0.423 & 95.55 \\
F3 & 8 & 0.45 & 0.10 & 0.45 & 12.48 & 0.190 & 91.59 \\
F4 & 12 & 0.45 & 0.10 & 0.45 & 13.44 & 0.241 & 94.44 \\
F5 & 8 & 0.25 & 0.50 & 0.25 & 74.34 & 0.414 & 97.98 \\
F6 & 12 & 0.25 & 0.50 & 0.25 & 111.40 & 0.452 & 97.44 \\
F7 & 8 & 0.40 & 0.50 & 0.10 & 31.64 & 0.235 & 97.63 \\
F8 & 12 & 0.40 & 0.50 & 0.10 & 40.63 & 0.413 & 95.07 \\
F9 & 8 & 0.30 & 0.20 & 0.50 & 20.29 & 0.258 & 96.94 \\
F10 & 12 & 0.30 & 0.20 & 0.50 & 38.50 & 0.919 & 95.40 \\
F11 & 10 & 0.20 & 0.30 & 0.50 & 123.00 & 0.848 & 97.66 \\
F12 & 10 & 0.45 & 0.30 & 0.25 & 20.57 & 0.355 & 96.61 \\
F13 & 10 & 0.30 & 0.10 & 0.60 & 75.02 & 0.192 & 93.57 \\
F14 & 10 & 0.40 & 0.40 & 0.20 & 27.02 & 0.291 & 97.28 \\
F15 & 10 & 0.30 & 0.30 & 0.40 & 34.6 & 0.532 & 93.31 \\
F16 & 10 & 0.30 & 0.30 & 0.40 & 44.27 & 0.507 & 95.78 \\
F17 & 10 & 0.30 & 0.30 & 0.40 & 25.33 & 0.209 & 95.96 \\
\hline
\end{tabular}

Table 6. The training and validation result of neural networks.

\begin{tabular}{lcccccc}
\hline \multirow{2}{*}{ Parameter } & \multicolumn{2}{c}{ Droplet size } & \multicolumn{2}{c}{ PDI } & \multicolumn{2}{c}{ Entrapment } \\
\cline { 2 - 6 } & Training & Validation & Training & Validation & Training & Validation \\
\hline$R^{2}$ & 0.989 & 0.999 & 0.875 & 0.891 & 0.907 & 1.000 \\
Root mean square error & 3.617 & 0.925 & 0.060 & 0.093 & 0.564 & 0.014 \\
Mean absolute deviation & 1.639 & 0.757 & 0.031 & 0.074 & 0.279 & 11.858 \\
Log-likelihood & 37.864 & 4.023 & -18.086 & -3.830 & -8.491 \\
Error sum of squares & 183.161 & 2.567 & 0.047 & 0.035 & 4.460 & 0.001 \\
Number of observations & 14 & 3 & 13 & 4 & 3 \\
\hline
\end{tabular}




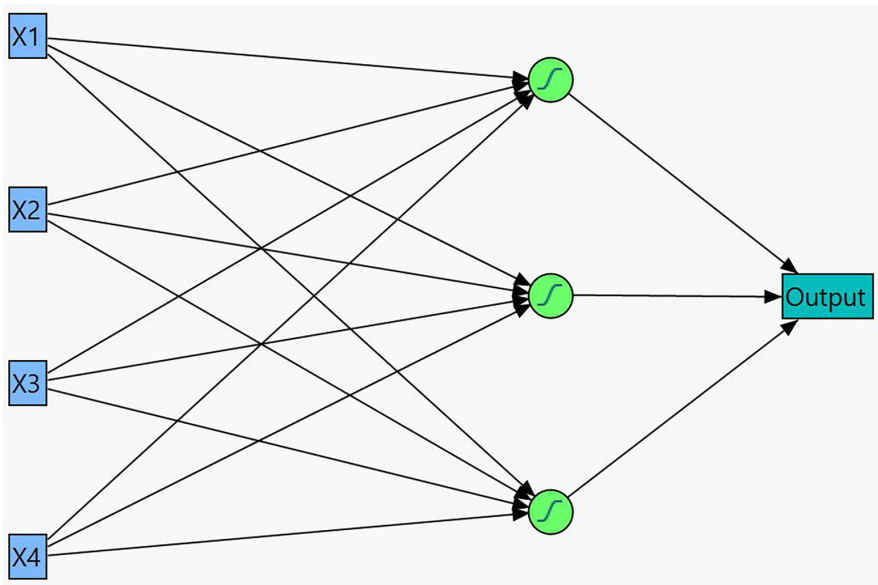

Figure 3. Diagram of neural network.

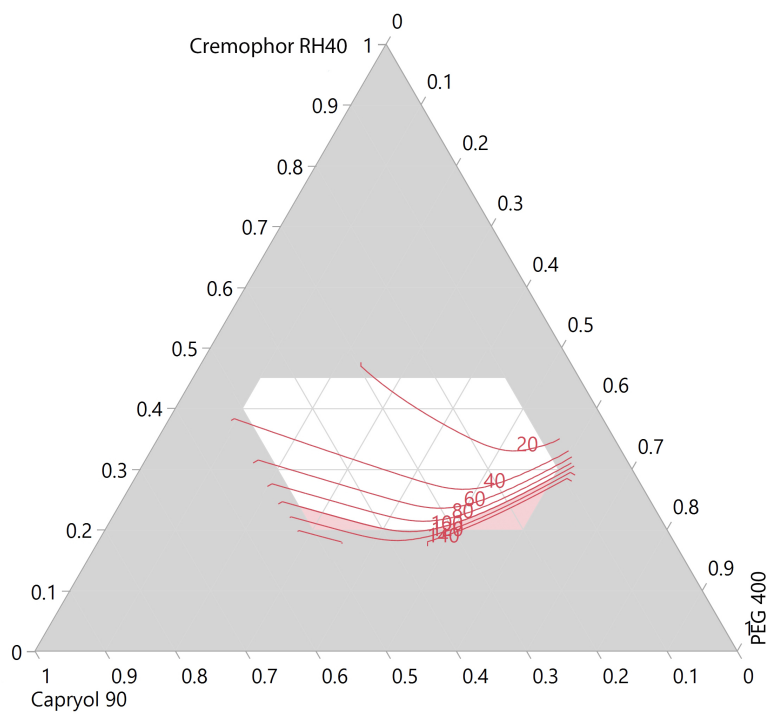

increased from $8 \%$ to $9 \%$, the drug entrapment decreased but when the percentage of Ros in the formulas increased from $9 \%$ to $12 \%$, the drug entrapment increases proportionally with the amount of drug substance.

Figure 6 shows the design space for optimizing the formula of Ros SNEDDS that was achieved by the superposition of three contour plots in Figures 4 and 5. It was clear that when the percentage of Ros increased from $8 \%$ to $12 \%$, the area for optimizing formula of Ros SNEDDS decreased.

When the percentage of Ros in the SNEDDS formula was at $8 \%$, the optimized formula of Ros SNEDDS could be determined by the area specified by the proportion of Cremophor RH40 in the range of $0.20-0.45$, Capryol 90 in the range of 0.10 0.43 and $0.48-0.50$, and PEG 400 in the range of $0.28-0.60$.

Meanwhile, at $10 \%$ of Ros in the SNEDDS formula, the area specified by the proportion of Cremophor RH40 in the range
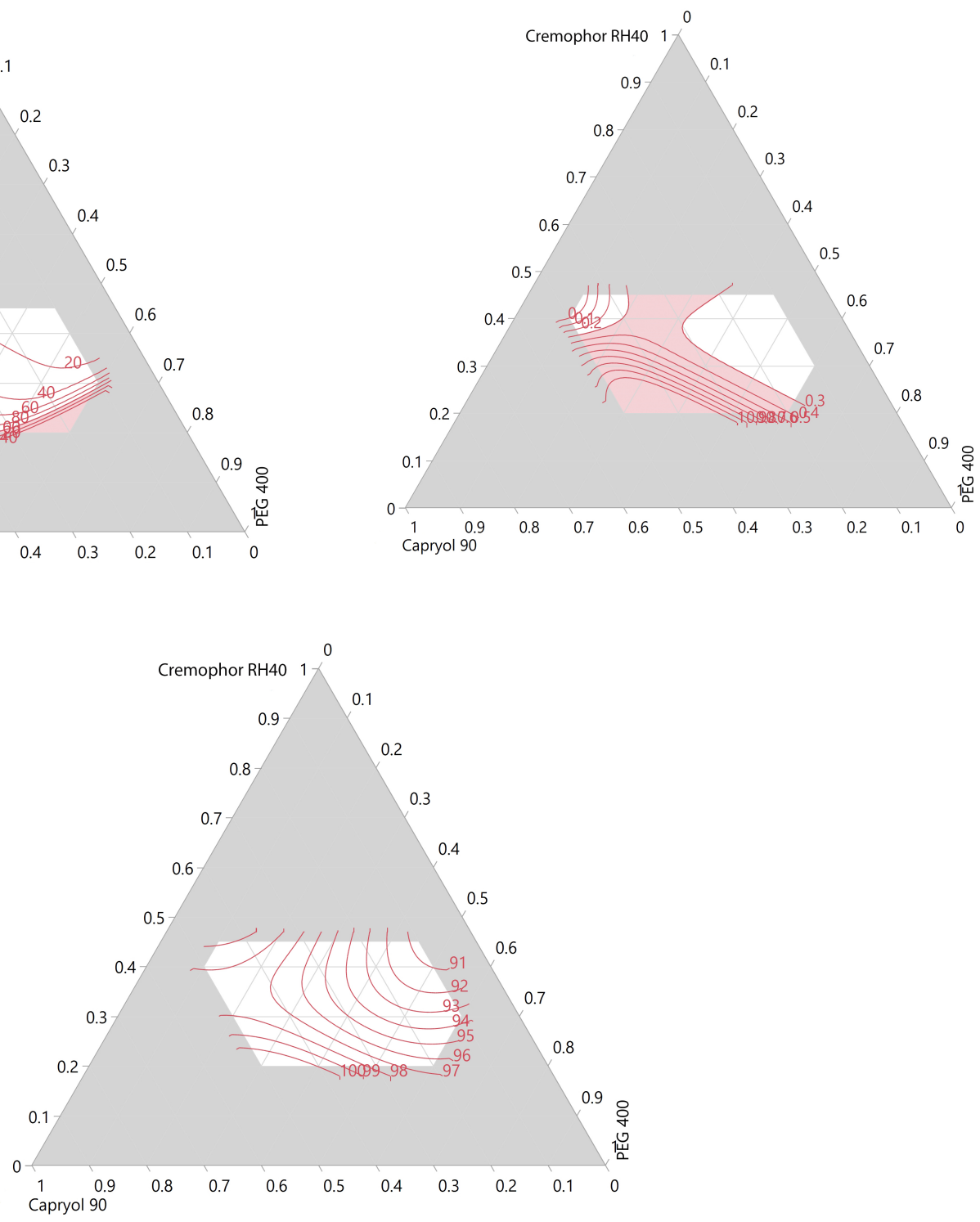

Figure 4. Contour plots showing the influences of SNEDDS components on the characteristics of Ros SNEDDS: droplet size (A), PDI (B), and Ros entrapment $(\mathrm{C})($ Ros percentage $=10 \%$; the white areas satisfy the optimum conditions of the output variables in Table 4$)$. 


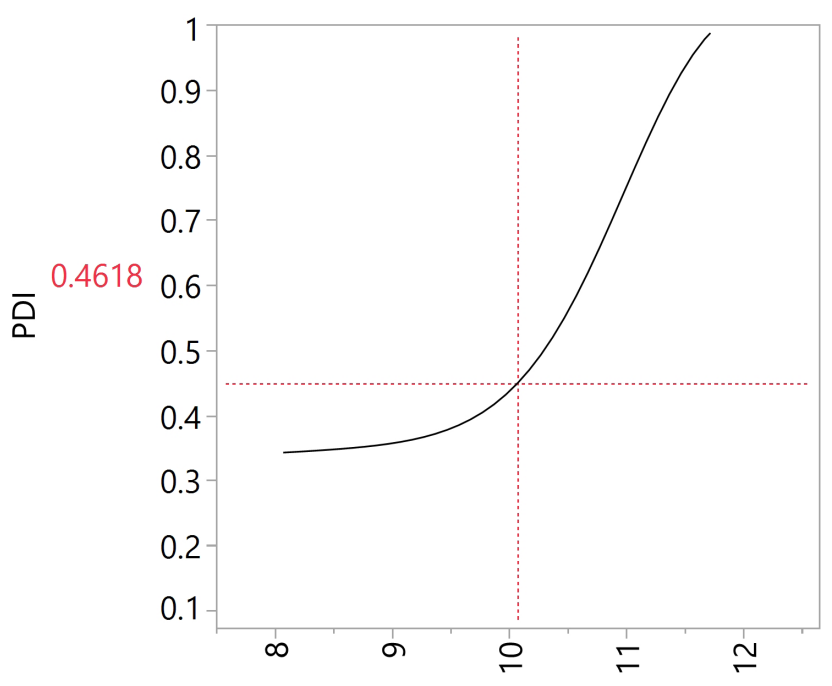

10

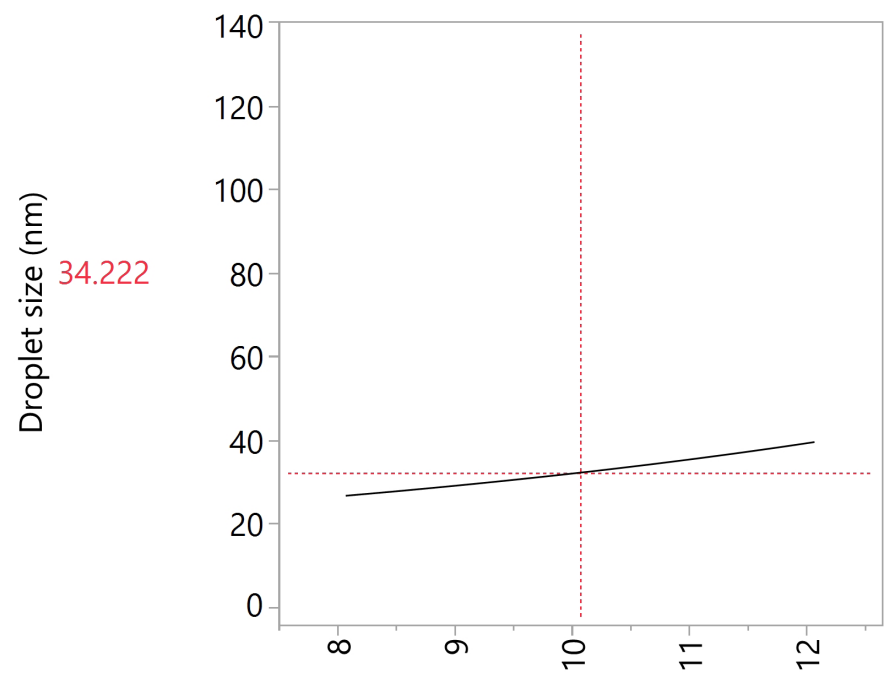

10

$\mathrm{X} 1(\%)$

$\mathrm{X} 1(\%)$

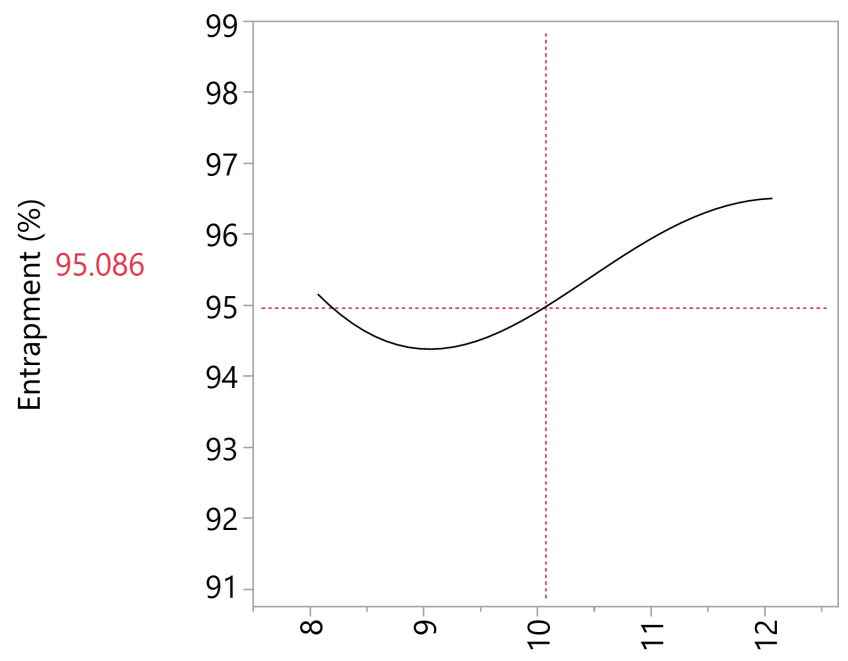

10

$\mathrm{X} 1(\%)$

Figure 5. Influences of the percentage of Ros (X1) on the characteristics of the SNEDDS: PDI (A), droplet size (B), and Ros entrapment (C) (proportion of Capryol $90=0.3$, Cremophor RH40 $=0.3$, and PEG $400=0.4$ ).

of $0.25-0.45$, Capryol 90 in the range of $0.10-0.31$ and $0.37-0.50$, and PEG 400 in the range of $0.10-0.20$ or $0.31-0.60$ can be used to determine the optimizing formula of Ros SNEDDS.

Unsurprisingly, at $12 \%$ of Ros in the SNEDDS formula, this area was smallest at the proportion of Cremophor RH40 in the range of $0.33-0.45$, Capryol 90 in the range of $0.10-0.18$ and $0.37-0.50$, and PEG 400 in the range of 0.37-0.53.

Normally, SNEDDS contains many components and the ratio among them has a strong influence on the characteristics of SNEDDS. Therefore, the formulation development of SNEDDS in a conventional way usually takes a lot of time and effort. In recent years, the artificial neural network has proved to be a very useful tool in establishing the relationship between input variables and product characteristics as well as optimizing formulas and manufacturing processes. In this study, the application of artificial neural networks in SNEDDS formulation development has shortened the time and reduced the research costs. It can be seen that the dependence of the most important characteristics of SNEDDS such as droplet size, PDI, and drug release on the system components can be modeled well by the artificial neural network. The droplet size of nanoemulsion, PDI, and Ros entrapment were affected and could be defined by synchronizing the proportion of 

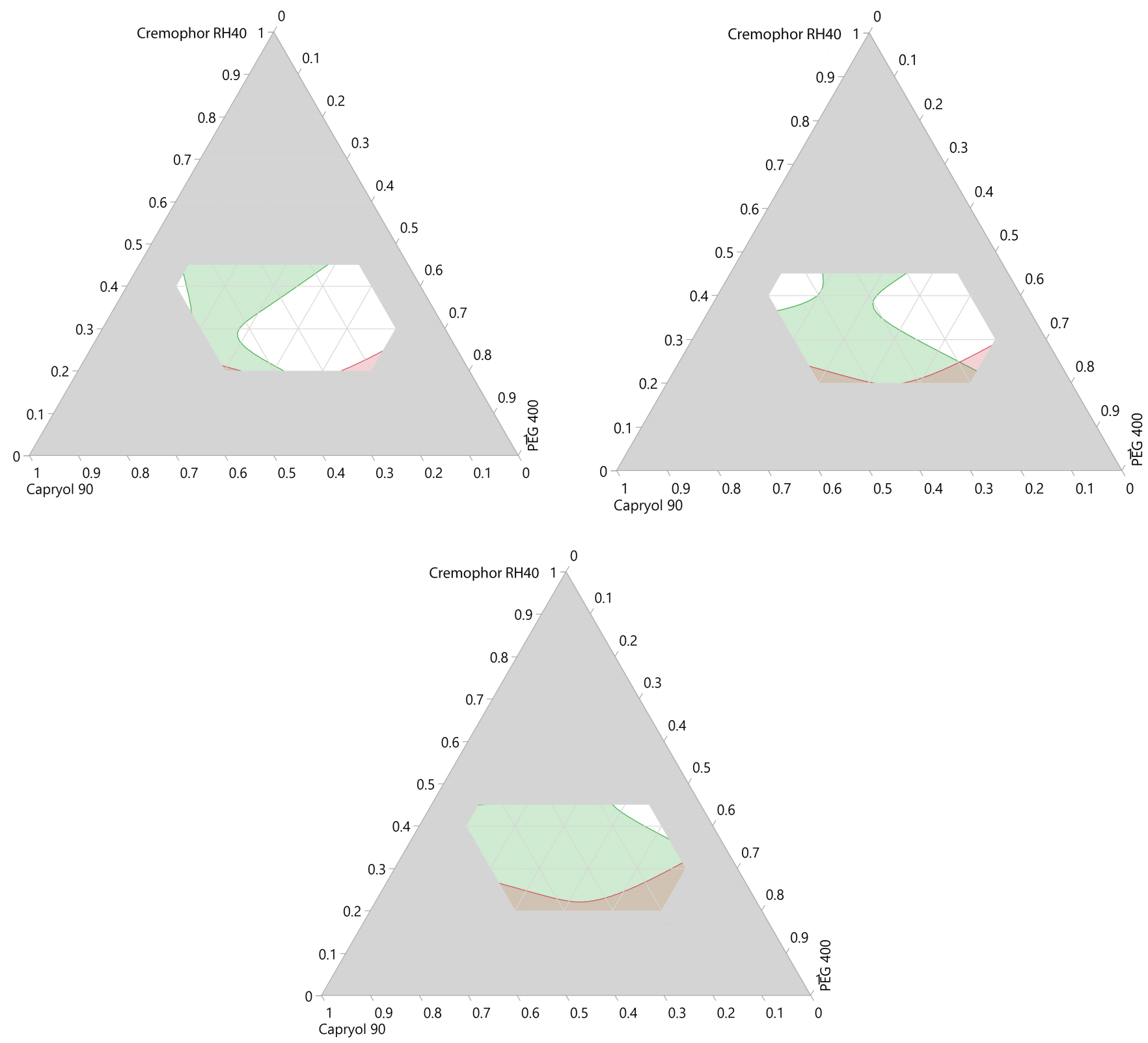

Figure 6. Design space (white areas) for optimizing formula of Ros SNEDDS with Ros percentage at $8 \%$ (A), $10 \%$ (B), and $12 \%$ (C) (red area: droplet size > 100 nm; green area: PDI $>0.3$; white area: optimum region [design space: droplet size (d.nm) $\leq 100$, PDI $\leq 0.3$, and Ros entrapment $\geq 90 \%$ in the whole experimental area].

excipients in the SNEEDS formulas. When the percentage of Ros in the SNEDDS formula was at $8 \%, 10 \%$, and $12 \%$, the optimized formula of Ros SNEDDS could be determined by different areas specified by the proportion of Cremophor RH40, Capryol 90, and PEG 400, respectively.

The optimization of the SNEDDS composition by defining the design space gives flexibility in formula development and process scale-up in the next research steps.

\section{Preparation and evaluation of physicochemical characteristics of the Ros SNEDDS}

Based on the design space for optimizing the formula of Ros SNEDDS, four optimum formulas of the SNEDDS containing Ros and their predicted characteristics were selected as shown in Table 7. The drug content of the Ros SNEDDS and physicochemical characteristics of the nanoemulsion including droplet size, PDI, Ros entrapment, and robustness to centrifugation are shown in Table 8.

After self-emulsification, Ros SNEDDS formed nanoemulsion with the average droplet sizes less than $100 \mathrm{~nm}$ and uniform droplet size distribution with PDI at less than 0.3 and showed robustness to centrifugation at 5,000 rpm for 30 minutes. Ros entrapment of all the SNEDDS samples was above $90 \%$.

Although there were deviations of droplet sizes and PDI from the prediction by the artificial neural network, our results also indicated that the physicochemical properties of nanoemulsion samples generated from the Ros SNEDDS after self-emulsification all met the initial optimization requirements: droplet size less than 
Table 7. The optimized formulas and predicted characteristics of Ros SNEDDS based on the artificial neural network.

\begin{tabular}{|c|c|c|c|c|}
\hline Samples & The optimized proportion of excipients & & Predicted characteristics & \\
\hline \multirow{4}{*}{ S1 } & Rosuvastatin calcium & $10.0 \mathrm{~g}$ & & \\
\hline & Cremophor RH40 & $42.0 \mathrm{~g}$ & Droplet size (d.nm) & 28.13 \\
\hline & Capryol 90 & $42.0 \mathrm{~g}$ & PDI & 0.186 \\
\hline & PEG 400 & $16.0 \mathrm{~g}$ & Ros entrapment (\%) & 98.23 \\
\hline \multirow{4}{*}{ S2 } & Rosuvastatin calcium & $10.0 \mathrm{~g}$ & & \\
\hline & Capryol 90 & $15.0 \mathrm{~g}$ & Droplet size (d.nm) & 14.46 \\
\hline & Cremophor RH40 & $45.0 \mathrm{~g}$ & PDI & 0.265 \\
\hline & PEG 400 & $40.0 \mathrm{~g}$ & Ros entrapment (\%) & 91.83 \\
\hline \multirow{4}{*}{ S3 } & Rosuvastatin calcium & $10.0 \mathrm{~g}$ & & \\
\hline & Capryol 90 & $24.0 \mathrm{~g}$ & Droplet size (d.nm) & 19.41 \\
\hline & Cremophor RH40 & $40.0 \mathrm{~g}$ & PDI & 0.271 \\
\hline & PEG 400 & $36.0 \mathrm{~g}$ & Ros entrapment (\%) & 93.67 \\
\hline \multirow{4}{*}{ S4 } & Rosuvastatin calcium & $11.0 \mathrm{~g}$ & & \\
\hline & Cremophor RH40 & $42.0 \mathrm{~g}$ & Droplet size (d.nm) & 28.00 \\
\hline & Capryol 90 & $42.0 \mathrm{~g}$ & PDI & 0.260 \\
\hline & PEG 400 & $16.0 \mathrm{~g}$ & Ros entrapment (\%) & 97.60 \\
\hline
\end{tabular}

Table 8. Physicochemical characteristics of optimized Ros SNEDDS samples $(n=3)$.

\begin{tabular}{lcccc}
\hline Characteristics & S1 & S2 & S3 & S4 \\
\hline Drug content (\%) & $9.01 \pm 0.20$ & $8.99 \pm 0.37$ & $9.05 \pm 2.80$ & $10.02 \pm 0.10$ \\
Droplet size (d.nm) & $22.41 \pm 0.50$ & $37.83 \pm 1.27$ & $55.30 \pm 0.18$ & $20.44 \pm 0.10$ \\
PDI & $0.153 \pm 0.005$ & $0.091 \pm 0.007$ & $0.115 \pm 0.005$ & $0.118 \pm 0.013$ \\
Ros entrapment (\%) & $94.72 \pm 0.11$ & $94.39 \pm 0.69$ & $90.77 \pm 1.44$ & $94.12 \pm 0.41$ \\
Robustness to centrifugation & $\begin{array}{c}\text { Homogeneous without } \\
\text { phase separation }\end{array}$ & $\begin{array}{c}\text { Homogeneous without } \\
\text { phase separation }\end{array}$ & $\begin{array}{c}\text { Homogeneous without } \\
\text { phase separation }\end{array}$ & $\begin{array}{c}\text { Homogeneous without } \\
\text { phase separation }\end{array}$ \\
\hline
\end{tabular}

$100 \mathrm{~nm}$ with PDI lower than 0.3, robustness to centrifugation at 5,000 rpm for 30 minutes, and Ros entrapment of all the SNEDDS samples above $90 \%$.

Our result also could be explained by understanding the chemical structure of rosuvastatin. Rosuvastatin is zwitterionic. The hydrophilic part consists of the acid functional group - $\mathrm{COOH}$ and the two functional groups of $-\mathrm{OH}$, while the lipophilic part consists of the methanesulfonamide pyrimidine ring and the phenyl group (Fong, 2014). This zwitterionic nature makes rosuvastatin compatible with oils, surfactants, and cosurfactants to form nanoemulsion with the droplet size below $100 \mathrm{~nm}$, and uniform droplet size distribution with PDI was always less than 0.3 .

In another perspective, several studies demonstrated that the dissolution medium in the gastrointestinal tract in the presence or absence of food did not significantly affect the droplet size, release rate, and absorption of active ingredients from SNEDDS (Kawakami et al., 2002). Nielsen et al. (2008) demonstrated that the oral bioavailability of probucol was not affected by food when the drug was formulated as a SNEDDS system; meanwhile, the results were opposite to the pellets containing free probucol.

The SNEDDS is a promising drug delivery system which could self-emulsify to form nanoemulsion in the digestive tract when exposed to gastrointestinal fluid (Bhanse and Shan, 2016; Date et al., 2010). It helps increasing solubility and improving the oral bioavailability of the drug substances. This method was successfully applied with other compounds in the statin group (Dixit and Nagarsenker, 2008; Elkadi et al., 2017; Kumar Mantri, 2012; Reddy, 2018).

\section{CONCLUSION}

We have successfully applied artificial neural network to optimize the formulation of Ros SNEDDS. Excipient mixtures containing Cremophor RH 40, Capryol 90, and PEG 400 as surfactant, oil, and cosurfactant, respectively, were chosen because of the high solubility of drug and the compatibility with rosuvastatin.

As the percentage of rosuvastatin calcium increased from $8 \%$ to $12 \%$, the area for optimizing the formula of Ros SNEDDS decreased. Various ranges of the excipients are defined based on the percentage of rosuvastatin calcium in the optimizing formula of Ros SNEDDS.

Our results also indicated that the prepared Ros SNEDDS samples, predicted by JMP 15 software after selfemulsification, possessed physicochemical properties that satisfied the optimization requirements: droplet size $\leq 100 \mathrm{~nm}$, PDI $\leq 0.3$, and Ros entrapment $\geq 90 \%$.

\section{ACKNOWLEDGMENTS}

The authors acknowledge the KC.10/16-20 Program of the Vietnam Ministry of Science and Technology for funding this research. They also acknowledge the Department of Pharmaceutics, 
Hanoi University of Pharmacy, Vietnam, and National Institute of Drug Quality Control, Vietnam, for their support to conduct this research.

\section{CONFLICT OF INTEREST}

The authors declare no conflicts of interest.

\section{REFERENCES}

Abo Enin HA. Self-nanoemulsifying drug-delivery system for improved oral bioavailability of rosuvastatin using natural oil antihyperlipdemic. Drug Dev Ind Pharm, 2015; 7:1047-56.

Alshora DH, Ibrahim MA, Elzayat E, Almeanazel, OT, Alanazi F. Rosuvastatin calcium nanoparticles: improving bioavailability by formulation and stabilization codesign. PLoS One, 2018; 13(7):e0200218.

Baek HH, Kim DH, Kwon SY, Rho SJ, Kim DW, Choi HG, Yong CS. Development of novel ibuprofen-loaded solid dispersion with enhanced bioavailability using cycloamylose. Arch Pharm Res, 2012, 4:683-9.

Bhanse ND, Shan CN. A review of research study on - self nanoemulsifying drug delivery system. J Pharm Sci Biosci Res, 2016; 5(6):621-7.

Chen H, Khemtong C, Yang X, Chang X, Gao J. Nanonization strategies for poorly water-soluble drugs. Drug Discov Today, 2011; $7-8: 354-60$.

Das SK, Kahali N, Bose A, Khanam J. Physicochemical characterization and in vitro dissolution performance of ibuprofenCaptisol ${ }^{\circledR}$ (sulfobutylether sodium salt of $\beta-C D$ ) inclusion complexes. J Mol Liq, 2018; 261:239-49.

DateAA, Desai N, Dixit R, Nagarsenker M. Self-nanoemulsifying drug delivery systems: formulation insights, applications and advances. Nanomedicine (Lond), 2010; 10:1595-616.

Dixit RP, Nagarsenker MS. Formulation and In vivo evaluation of self-nanoemulsifying granules for oral delivery of a combination of rzetimibe and simvastatin. Drug Dev Ind Pharm, 2008; 12:1285-96.

Elkadi S, Elsamaligy S, Al-Suwayeh S, Mahmoud H. The development of self-nanoemulsifying liquisolid tablets to improve the dissolution of simvastatin. AAPS PharmSciTech, 2017; 7:2586-97.

Fong CW. Statins in therapy: understanding their hydrophilicity, lipophilicity, binding to 3-hydroxy-3-methylglutaryl-CoA reductase, ability to cross the blood brain barrier and metabolic stability based on electrostatic molecular orbital studies. Eur J Med Chem, 2014; 85:661-74.

Gabr MM, Mortada SM, Sallam M A. Carboxylate cross-linked cyclodextrin: a nanoporous scaffold for enhancement of rosuvastatin oral bioavailability. Eur J Pharm Sci, 2018; 111:1-12.

Hong JY, Kim JK, Song YK, Park JS, Kim CK. A new selfemulsifying formulation of itraconazole with improved dissolution and oral absorption. J Control Release, 2006; 2:332-8.

Kamble PR, Shaikh KS, Chaudhari PD. Application of liquisolid technology for enhancing solubility and dissolution of rosuvastatin. Adv Pharm Bull, 2014; 2:197-204.

Kapse Vidya N, Chemate SZ, Dharashive VM. Formulation development and solubility enhancement of rosuvastatin calcium tablet prepared by complexation with $\beta$-cyclodextrin by kneading method. Int $\mathrm{J}$ Pharm Sci Res, 2016; 18:4882-92.

Karasulu H, Gundogdu E. Enhancing solubility and bioavailability of rosuvastatin into self nanoemulsifying drug delivery system. Curr Drug Deliv, 2018; 7:1072-82.

Kassem AM, Ibrahim HM, Samy AM. Development and optimisation of atorvastatin calcium loaded self-nanoemulsifying drug delivery system (SNEDDS) for enhancing oral bioavailability: in vitro and in vivo evaluation. J Microencapsul, 2017; 3:319-33.

Kawabata Y, Wada K, Nakatani M, Yamada S, Onoue S. Formulation design for poorly water-soluble drugs based on biopharmaceutics classification system: basic approaches and practical applications. Int J Pharm, 2011; 1:1-10.

Kawakami K, Yoshikawa T, Moroto Y, Kanaoka E, Takahashi K, Nishihara Y, Masuda K. Microemulsion formulation for enhanced absorption of poorly soluble drugs: I. Prescription design. J Control Release, 2002; 1-2:65-74.

Krishnamoorthy V, Prasad VPR, Sen S. Formulation and evaluation of risperidone-mannitol solid dispersions. Farmacia, 2012 6:877-94.

Kumar Katual M, Singh GL, Harikumar S. Solubility enhancement of poorly aqueous soluble novel drug using natural gum as carriers. Panacea J Med Sci, 2020; 3:104-16.

Kumar Mantri S. Development and characterization of selfnanoemulsifying drug delivery systems (SNEDDS) of atorvastatin calcium. Curr Drug Deliv, 2012; 2:182-96.

Li J, Yang $\mathrm{M}, \mathrm{Xu}$ W. Development of novel rosuvastatin nanostructured lipid carriers for oral delivery in an animal model. Drug Des Devel Ther, 2018; 12:2241-8.

Luvai A, Mbagaya W, Hall AS, Barth JH. Rosuvastatin: a review of the pharmacology and clinical effectiveness in cardiovascular disease Clin Med Insights Cardiol, 2012; 6:17-33.

Mahmoud H. Design and optimization of self-nanoemulsifying drug delivery systems of simvastatin aiming dissolution enhancement. Afr J Pharm Pharmacol, 2013; 22:1482-500.

Mahmoud H, Suwayeh SA, Elkadi S. Design and optimization of self-nanoemulsifying drug delivery systems of simvastatin aiming dissolution enhancement. Afr J Pharm Pharmacol, 2013; 22:1482-500.

Nielsen FS, Petersen KB, Müllertz A. Bioavailability of probucol from lipid and surfactant based formulations in minipigs: influence of droplet size and dietary state. Eur J Pharm Biopharm, 2008; 2:553-62.

Olsson AG, McTaggart F, Raza A. Rosuvastatin: a highly effective new HMG-CoA reductase inhibitor. Cardiovasc Drug Rev, 2006; 4:303-28.

Palani K, Christoper PGV, Kesavan SK. Enhancement of rosuvastatin calcium bioavailability applying nanocrystal technology and in-vitro, in-vivo evaluations. Asian J Pharm Clin Res, 2015; 2:88-92.

Reddy MS. Formulation and In vitro characterization of solidself nanoemulsifying drug delivery system of atorvastatin calcium. Asian J Pharm, 2018; 4:991-9.

Rizwanullah M, Amin S, Ahmad J. Improved pharmacokinetics and antihyperlipidemic efficacy of rosuvastatin-loaded nanostructured lipid carriers. J Drug Target, 2017; 1:58-74.

Sarfraz RM, Ahmad M, Mahmood A, Minhas MU, Yaqoob A. Development and evaluation of rosuvastatin calcium based microparticles for solubility enhancement: an In vitro Study. Adv Polym Technol, 2017; 4:433-41.

Schachter M. Chemical, pharmacokinetic and pharmacodynamic properties of statins: an update. Fundam Clin Pharmacol, 2005; 1:117-25.

Verma S, Singh SK, Verma PRP, Ahsan MN. Formulation by design of felodipine loaded liquid and solid self nanoemulsifying drug delivery systems using Box-Behnken design. Drug Dev Ind Pharm, 2014; $10: 1358-70$.

Wang L, Dong J, Chen J, Eastoe J, Li X. Design and optimization of a new self-nanoemulsifying drug delivery system. J Colloid Interface Sci, $2009 ; 2: 443-8$

\section{How to cite this article:}

Vu GTT, Phan NT, Nguyen HT, Nguyen HC, Tran YTH, Pham TB, Nguyen LT, Nguyen HD. Application of the artificial neural network to optimize the formulation of self-nanoemulsifying drug delivery system containing rosuvastatin. J Appl Pharm Sci, 2020; 10(09):001-011. 